\title{
Lorentz and CPT violation in the Standard-Model Extension
}

\author{
Ralf Lehnert
}

Published online: 19 February 2013

\begin{abstract}
Lorentz and CPT invariance are among the symmetries that can be investigated with ultrahigh precision in subatomic physics. Being spacetime symmetries, Lorentz and CPT invariance can be violated by minuscule amounts in many theoretical approaches to underlying physics that involve novel spacetime concepts, such as quantized versions of gravity. Regardless of the underlying mechanism, the low-energy effects of such violations are expected to be governed by effective field theory. This talk provides a survey of this idea and includes an overview of experimental efforts in the field.
\end{abstract}

Keywords Lorentz-symmetry violation $\cdot$ CPT-symmetry violation · quantum gravity

PACS 11.30.Er $\cdot$ 32.10.Fn $\cdot 04.30 .-\mathrm{w}$

\section{Introduction}

At present, substantial research efforts are directed at uncovering fundamental physics underlying the Standard Model (SM) and General Relativity (GR). While there are a number of purely theoretical approaches to this subject, phenomenological progress in this field is inhibited by the expected Planck suppression of experimental signatures associated with these types of new physics.

Within this context, small departures from Lorentz and CPT invariance are presently being considered promising candidate signatures: many theoretical

Ralf Lehnert

Indiana University Center for Spacetime Symmetries, Bloomington, IN 47405, USA

Tel.: +1-812-855-6190

Fax: +1-812-855-5533

E-mail: ralehner@indiana.edu 
ideas for underlying physics can lead to Lorentz- and CPT-symmetry violations [1, and current technology allows numerous types of Planck-sensitivity tests of these symmetries in a broad range of physical systems [2].

For the comparison of different measurements in this field and the identification of future tests, a theoretical description of small departures from Lorentz and CPT invariance is desirable. A widely employed framework for this purpose, called the Standard-Model Extension (SME), is based on effective field theory [3]. The SME framework does not represent a particular theoretical approach to fundamental physics underlying the SM and GR; it rather describes the potential Lorentz- and CPT-violating effects of such theoretical ideas at presently attainable energies in a general and practically model-independent way.

This work describes the subject in a top-down manner. Section 2 lists various mechanisms for Lorentz- and CPT-symmetry breaking in underlying physics at high energies. In Sec. 3, a brief review of the main ideas behind the construction and the application of the SME framework is given. Some experimental searches for deviations from Lorentz and CPT invariance are described in Sec. 4 .

\section{Mechanisms for deviations from Lorentz and CPT invariance}

Although most approaches to underlying physics are based on scenarios with Lorentz- and CPT-symmetric dynamics, they can nevertheless lead to ground states in which these symmetries are - at least partially - absent. The mechanisms triggering these symmetry violations represent a key motivation for Lorentz and CPT tests. For this reason, we devote this section to an (incomplete) list of sample mechanisms for Lorentz and CPT breakdown. More details about the individual mechanisms can be found in Ref. 11.

Spontaneous Lorentz- and CPT-invariance violation. - Spontaneous symmetry breaking (SSB) in general is well established in physics and theoretically very attractive. The basic idea behind SSB is that the lowest-energy state of the system, which is usually taken to be the vacuum, requires the value of a suitable field to be non-vanishing. Such non-zero vacuum expectation values (VEVs) often do not exhibit all dynamical symmetries of the system, and they lead to the main observable imprints from SSB. In the case of Lorentz and CPT breaking, these VEVs are non-zero vector or tensor fields. In the flat-spacetime limit, these VEVs are typically taken as constant, which clearly shows the selection of preferred directions and thus the loss of exact Lorentz and $\mathrm{CPT}$ invariance. A similar, slightly more complex reasoning also applies in gravitational contexts. It is difficult to fit the interactions required for the spontaneous violation of Lorentz and CPT invariance into the framework of conventional renormalizable gauge theories. However, string field theory provides a natural setting for such interactions.

Spacetime-dependent scalars. - A number of candidate theories for underlying physics accommodate scalar fields other than the SM Higgs. Note also 
that various astrophysical measurements can for example be explained with new scalar fields. In cosmological contexts, such scalar fields often acquire a non-vanishing global value with a time evolution driven by the expansion of the universe. Such a background clearly violates spacetime translation invariance. Since translations and Lorentz transformations are linked in the Poincaré group, it is natural to expect that this breaking of translation invariance will also lead to a violation of Lorentz (and possibly CPT) symmetry. This is intuitively reasonable because a varying scalar possesses a non-trivial gradient, and this gradient selects a preferred direction.

Non-commutative field theory.-In this approach to underlying physics, the basic idea is that coordinates are no longer real numbers. Instead, they are operators that satisfy non-trivial commutation relations. For instance, the relation $\left[x^{\mu}, x^{\nu}\right]=i \theta^{\mu \nu}$, where $\theta^{\mu \nu} \neq 0$ is constant, is typically employed as an example. Clearly, $\theta^{\mu \nu}$ is the distinguishing feature of non-commutative field theory, so it must govern certain phenomenological effects. But as in the above two examples, a constant non-dynamical $\theta^{\mu \nu}$ selects preferred directions in spacetime, so that non-commutative models generically violate Lorentz symmetry.

Loop quantum gravity.-Another popular approach to physics beyond the $\mathrm{SM}$ and GR is loop quantum gravity, which considers a version of GR that is quantized in a particular way. Some semiclassical analyses of loop quantum gravity have established various results that are incompatible with exact Lorentz invariance. For instance, under certain reasonable physical assumptions both fermions and electrodynamics receive low-energy loop-quantumgravity corrections that are associated with preferred directions. As for the previous mechanisms, such preferred directions represent a violation of Lorentz symmetry.

\section{The SME test framework}

Given the multitude of sample mechanisms for Lorentz- and CPT-symmetry breaking listed in the previous section, the question arises how to describe general departures from these symmetries at low energies. This question is exacerbated by the fact that for some of these mechanisms the direct extraction of the low-energy limit is presently unclear. To circumvent these issues, it is advantageous to construct a suitable test framework by hand. Such a test framework should be as general as possible while maintaining desirable physical principles. These ideas have led to the establishment of the SME mentioned in the introduction [3]. What follows is a brief review of the main ingredients for the construction of the SME framework.

One of these ingredients is the feature common to all of the above sample mechanisms for Lorentz- and CPT-invariance violation: the emergence of preferred directions in the vacuum, which are responsible for the symmetry breakdown. For this reason, the departures from Lorentz and CPT invariance 
in the SME are parametrized by non-dynamical external vector or tensor fields $b^{\mu}, c^{\mu \nu}$, etc.

A second ingredient is effective field theory (EFT). This framework is widely used with great success in various areas of physics including elementaryparticle, nuclear, and condensed-matter physics; it provides a general and flexible description of dynamical systems with large numbers of degrees of freedom. It is therefore a reasonable assumption that the low-energy limit of potential violations of Lorentz and CPT invariance arising from underlying physics can be described within EFT. This leads to a lagrangian formulation of the SME as an EFT.

A third key ingredient is coordinate independence: although Lorentz and CPT symmetry are broken, it should still be possible to select any suitable reference frame for the mathematical description of physical laws. In other words, coordinates are a product of human thought and should not acquire physical significance. Coordinate independence is guaranteed if physics is formulated in terms geometrical quantities, such as scalars, vectors, tensors, and spinors.

These considerations lead to the following general structure for the SME Lagrangian $\mathcal{L}_{\mathrm{SME}}$ :

$$
\mathcal{L}_{\mathrm{SME}}=\mathcal{L}_{\mathrm{SM}}+\mathcal{L}_{\mathrm{EH}}+\delta \mathcal{L}_{\mathrm{SME}}
$$

where $\mathcal{L}_{\mathrm{SM}}$ and $\mathcal{L}_{\mathrm{EH}}$ are the ordinary Standard-Model and Einstein-Hilbert contributions, and $\delta \mathcal{L}_{\mathrm{SME}}$ contains small Lorentz- and CPT-violating corrections constructed according the above ingredients:

$$
\delta \mathcal{L}_{\mathrm{SME}}=-\bar{\psi} b^{\mu} \gamma_{5} \gamma_{\mu} \psi+i \bar{\psi} c^{\mu \nu} \gamma_{\mu} \partial_{\nu} \psi+\ldots
$$

Here, $b^{\mu}$ and $c^{\mu \nu}$ are the aforementioned examples for preferred directions generated by underlying physics. Within the context of the SME, they represent coefficients controlling the type and extent of Lorentz-symmetry breaking. We remark that the $b^{\mu}$ coefficient also governs certain types of CPT violation, while $c^{\mu \nu}$ is CPT even. Various theoretical investigations have been performed within the SME [4, but none have found theoretical inconsistencies thus far.

In an EFT, one typically expects the mass-dimension three and four operators to dominate at low energies. The restriction of the SME to these operators is sometimes called the minimal SME, but higher-dimensional operators have been considered as well [5].

\section{Experimental searches for Lorentz- and CPT-symmetry violation}

Lorentz and CPT invariance underpin the behavior of numerous physical systems, so that these symmetries can be tested in a correspondingly broad range of experiments [2]. The SME test framework discussed in the previous section can be used to make predictions and compare results for virtually all such tests. Examples for Lorentz- and CPT-symmetry measurements include tests with cosmic radiation 6], particle colliders [7], resonance cavities 8, neutrinos 9, and precision spectroscopy [10]. 
One might expect that effects from underlying physics - such as Lorentz and CPT breakdown - become more pronounced as the energy scale is increased because more degrees of freedom (including those from the underlying theory) can be excited. However, low-energy tests typically offer exquisite precisions that can more than offset the expected suppression of the effect. For this reason, low-energy physics offers excellent prospects in the search for Planck-suppressed deviations from Lorentz and CPT invariance.

Many simple low-energy systems are bound states. A key SME prediction for bound states is the shift of energy levels. Since these can be measured with ultrahigh precision, they are suitable for Lorentz and CPT tests. One class of experiments in this context compares the energy levels of matter and antimatter bound states. An example for planned measurements of this type is antihydrogen spectroscopy [11. These tests would be primarily sensitive to those types of Lorentz breaking that also involve CPT violation. Another class of experiments searches for sidereal variations in the spacing of energy levels. The idea here is that Lorentz invariance includes rotation symmetry, so that there might arise effects from the rotation of the laboratory around the Earth's axis 12. Placing the experiment on a turn table has also been used recently to search for anisotropies. A third class of tests seeks to measure the invariance under boosts by studying the behavior of the energy levels under velocity changes. Many experiments in this context exploit the motion of the Earth around the Sun [13. But space-based tests on satellites offer a viable alternative in this context.

Various free-particle physical systems can also be considered to be at comparatively low energies. Examples in this context involve measurements of the muon magnetic moment at a storage ring. Such an experiment is also affected by SME coefficients, and the corresponding data has previously been employed to place constraints on Lorentz- and CPT-symmetry violation [14. Another recent approach in this context searches for effects in the weak interaction by observing the decay of ${ }^{80} \mathrm{Rb}$ or ${ }^{20} \mathrm{Na}$ as a function of sidereal time. The first results for this test are expected soon [15].

Acknowledgements The author wishes to thank the organizers for arranging this stimulating meeting and for the invitation to participate. This work is supported by the Indiana University Center for Spacetime Symmetries.

\section{References}

1. See, e.g., V.A. Kostelecký and S. Samuel, Phys. Rev. D 39, 683 (1989); J. Alfaro, H.A. Morales-Técotl, and L.F. Urrutia, Phys. Rev. Lett. 84, 2318 (2000); S.M. Carroll et al., Phys. Rev. Lett. 87, 141601 (2001); J.D. Bjorken, Phys. Rev. D 67, 043508 (2003); V.A. Kostelecký et al., Phys. Rev. D 68, 123511 (2003); O. Bertolami et al., Phys. Rev. D 69, 083513 (2004).

2. V.A. Kostelecký and N. Russell, Rev. Mod. Phys. 83, 11 (2011).

3. D. Colladay and V.A. Kostelecký, Phys. Rev. D 58, 116002 (1998); V.A. Kostelecký and R. Lehnert, Phys. Rev. D 63, 065008 (2001); V.A. Kostelecký, Phys. Rev. D 69 , 105009 (2004). 
4. See, e.g., R. Jackiw and V.A. Kostelecký, Phys. Rev. Lett. 82, 3572 (1999); V.A. Kostelecký et al., Phys. Rev. D 65, 056006 (2002); B. Altschul and V.A. Kostelecký, Phys. Lett. B 628, 106 (2005); R. Lehnert, Phys. Rev. D 68, 085003 (2003); J. Math. Phys. 45, 3399 (2004); Phys. Rev. D 74, 125001 (2006); Rev. Mex. Fís. 56 (6), 469 (2010); A.J. Hariton and R. Lehnert, Phys. Lett. A 367, 11 (2007); D. Colladay and P. McDonald, Phys. Rev. D 77, 085006 (2008); Phys. Rev. D D79, 125019 (2009).

5. R.C. Myers and M. Pospelov, Phys. Rev. Lett. 90, 211601 (2003); C.M. Reyes et al., Phys. Rev. D 78, 125011 (2008); V.A. Kostelecký and M. Mewes, Phys. Rev. D 80 015020 (2009); Phys. Rev. D 85, 096005 (2012); M. Cambiaso et al., Phys. Rev. D 85, 085023 (2012); G. Rubtsov et al., Phys. Rev. D 86, 085012 (2012).

6. See, e.g., V.A. Kostelecký and M. Mewes, Phys. Rev. Lett. 87, 251304 (2001); Phys. Rev. D 66, 056005 (2002); R. Lehnert and R. Potting, Phys. Rev. Lett. 93, 110402 (2004); Phys. Rev. D 70, 125010 (2004); B.D. Altschul, Astropart. Phys. 28, 380 (2007); Phys. Rev. D 78, 085018 (2008).

7. See, e.g., M.A. Hohensee et al., Phys. Rev. Lett. 102, 170402 (2009); Phys. Rev. D 80 036010 (2009); G. Amelino-Camelia et al., Eur. Phys. J. C 68, 619 (2010); V. Gharibyan, Phys. Lett. B 611, 231 (2005); J.-P. Bocquet et al. [GRAAL Collaboration], Phys. Rev. Lett. 104, 241601 (2010); B.D. Altschul, Phys. Rev. D 80, 091901 (2009); Phys. Rev. D 84, 076006 (2011).

8. H. Müller et al., Phys. Rev. D 68, 116006 (2003); Ch. Eisele et al., Phys. Rev. Lett. 103, 090401 (2009); M.E. Tobar et al., Phys. Rev. D 80, 125024 (2009).

9. T. Katori et al., Phys. Rev. D 74, 105009 (2006); J.S. Díaz et al., Phys. Rev. D 80, 076007 (2009); V. Barger et al., Phys. Rev. D 84, 056014 (2011); J.S. Díaz and V.A. Kostelecký, Phys. Rev. D 85, 016013 (2012).

10. R. Bluhm et al., Phys. Rev. D 57, 3932 (1998); H. Dehmelt et al., Phys. Rev. Lett. 83, 4694 (1999); R. Mittleman et al., Phys. Rev. Lett. 83, 2116 (1999); G. Gabrielse et al., Phys. Rev. Lett. 82, 3198 (1999); D. Bear et al., Phys. Rev. Lett. 85, 5038 (2000); R. Lehnert and I. Shimamura, J. Plasma Fusion Res. 80, 1006 (2004); P. Wolf et al., Phys. Rev. Lett. 96, 060801 (2006); I. Altarev et al., Phys. Rev. Lett. 103, 081602 (2009); Europhys. Lett. 92, 51001 (2010).

11. See, e.g., R. Bluhm et al., Phys. Rev. Lett. 82, 2254 (1999).

12. See, e.g., V.W. Hughes et al., Phys. Rev. Lett. 87, 111804 (2001).

13. See, e.g., F. Canè et al., Phys. Rev. Lett. 93, 230801 (2004).

14. See, e.g., G.W. Bennett et al. [Muon (g-2) Collaboration], Phys. Rev. Lett. 100, 091602 (2008).

15. S.E. Müller, Hyperfine Interact. 215, no. 1-3, 31 (2013). 